\title{
Falsificación de marcas, competencia desleal y sanciones de la organización mundial del comercio.
} Trademark Counterfeiting, Unfair Competition and World Trade Organization
Sanctions.

\author{
Ing. Pedro Ufredo León Cedeño ${ }^{{ }^{*}}$, Lic. Ítalo Omar Martillo Pazmiño ${ }^{2}$
}

1* Magister en Administración de Empresas. Universidad Estatal de Guayaquil, Guayaquil, Ecuador. Email: pedro.leonce@ug.edu.ec ORCID: https://orcid.org/0000-0002-4510-9216

2. Magister en Administración de Empresas Mención en Negocios Internacionales. Universidad de Guayaquil, Guayaquil, Ecuador. Email: italo.martillop@ug.edu.ec ORCID: https://orcid.org/0000-0003-3762-221X

Destinatario:pedro.leonce@ug.edu.ec

Recibido: 09/Agosto/2021

Aceptado: 10/Septiembre/2021

Publicado: 29/Octubre/2021

\begin{abstract}
Como citar: León Cedeño , P. U., \& Martillo Pazmiño , Ítalo O. (2020). Falsificación de marcas, competencia desleal y sanciones de la organización mundial del comercio. E-IDEA Journal of Business Sciences, 3(13), 12-26. https://doi.org/10.53734/eidea.vol3.id141
\end{abstract}

\begin{abstract}
Resumen: El comercio es una de las actividades más estratégicas en el mundo actual; ya que genera fuentes de empleo directos e indirectos, ingresos, se incentiva el gasto, consumo y tributos, sin embargo el proceso no siempre funciona en la legalidad, por ello el tema de investigación acerca de la falsificación de marcas, competencia desleal y actuación de la organización mundial del comercio para prevenir la falsificación, en el método se utiliza la descripción, investigación documental con el uso de técnicas como análisis de contenido y análisis de documentos, informes y acuerdos de la Organización Mundial del Comercio. Los resultados muestran y comparan cifras y porcentajes, se concluye con las acciones que realizan varios Estados para controlar dichas actividades, la protección de la propiedad intelectual es insuficiente; la tecnología contribuye a que realizar copias de productos sea una actividad fácil de realizar con escaso control.
\end{abstract}

Palabras clave: Marcas, falsificación de marcas, propiedad intelectual, propiedad industrial patente, competencia desleal.

Abstract: Trade is one of the most strategic activities in today's world; since it generates direct and indirect sources of employment, income, spending, consumption and taxes are encouraged, however the process does not always work legally, therefore the research topic on trademark counterfeiting, unfair competition and the performance of the world trade organization to prevent counterfeiting, the method uses description, documentary research with the use of techniques such as content analysis and analysis of documents, reports and agreements of the World Trade Organization. The results show and compare figures and percentages, it is concluded with the actions carried out by various States to control said activities, the protection of intellectual property is insufficient; technology helps make product copies easy to do with little control.

Keywords: Trademarks, trademark counterfeiting, intellectual property, patent industrial property, unfair competition. 


\section{INTRODUCCIÓN}

$\mathrm{E}$

l el mundo actual las operaciones de compra-venta expresadas en comercio implican uno de los factores estratégicos para garantizar ingreso, empleo, consumo, distribución de bienes y servicios; sin embargo en la dinámica propia del mercado las condiciones cambian en diferentes contextos, así la competencia no siempre es leal, por el contrario se evidencia la competencia desleal a través de actividades como la falsificación de marcas el cual constituye delito que pocas veces es reconocido y aceptado como tal.

En la búsqueda del tema de investigación, en concordancia con las características del método científico se ha elegido como título del artículo: falsificación de marcas, competencia desleal y actuación de la Organización Mundial del Comercio para evitar la proliferación de dichos artículos por el mundo, con el uso del método descriptivo en concordancia con la investigación documental en el cual se utilizan la técnicas de análisis de contenido de fuentes secundarias que consiste en la interpretación y análisis de documentos, informes y acuerdos inherentes a la Organización Mundial del Comercio.

La originalidad y registro formal, de la marca como identidad y símbolo de productos está protegido en la figuras de la propiedad intelectual y propiedad industrial, convenios cuya creación proviene de finales del siglo XVIII con los Convenios de París y Berna, las ideas son el primer antecedente de una creación científica, artística o literaria con el potencial de mejorarlos para fabricar productos dirigidos a satisfacer las expectativas de los usuarios; diferentes organismos como la Unión Europea y la Organización Mundial del Comercio han creado departamentos con el objetivo de vigilar y controlar la falsificación de marcas; sin embargo ello no es suficiente, la propia innovación tecnológica se han convertido en un factor contradictorio que propicia la falsificación.

El artículo se divide en tres apartados: Definición de Marca, Valor de la Marca y Funciones, el segundo apartado Falsificación de Marcas y Convenio de Propiedad Industrial, por último, los resultados en el cual se muestran y comparan cifras, porcentajes, países y lugares en los cuales se ha destacado mayor actividad de falsificación de firmas además de las acciones que realizan varios Estados para controlar dichas actividades.

\section{METODOLOGÍA}

La palabra Método significa el procedimiento ordenado y sistemático con la finalidad de obtener resultados, por ello se asocia frecuentemente a la ciencia; por su propia naturaleza el estudio observación, análisis y formulación de resultados acerca de un hecho $\mathrm{u}$ objeto de estudio requiere el cumplimiento de una serie de requisitos inherentes a objetividad, racionalidad, utilidad y exactitud entre otros. Por ello el método científico se apoya en diferentes categorías, tipos o diseños de investigación en concordancia con el 


\section{E-IDEA}

R E V I S T A

J OURNAL OF BUSINESS SCIENCES

contenido y dimensión del estudio, cabe destacar que uno de los métodos consiste en formular una premisa inicial, como se indicará en el apartado de Desarrollo.

Para lograr responder a dicha interrogante, se considera pertinente la aplicación del tipo de investigación descriptiva porque aporta la secuencia coherente de la situación que se explica a través de la demostración de método científico, lo cual se realiza mediante el análisis de contenido de los documentos de diferentes fuentes, textos, documentos con el propósito de establecer la información trascendente para comprobar el tema de estudio, Sampieri, Fernández y Baptista (2019) explican:

Con los estudios descriptivos se busca especificar las propiedades, las características y los perfiles de personas grupos, comunidades, procesos, objetos o cualquier otro fenómeno que se someta a un análisis... únicamente pretenden medir o recoger información de manera independiente o conjunta sobre los conceptos o variables a las que se refieren (pág. 92)

Por consiguiente, es un análisis exhaustivo, en el cual se recolecta información de fuentes primarias se compara y explica es una significativa estrategia que aporta al investigador la facilidad de hallar características, y vinculaciones entre las variables así es posible comprobar la concordancia, coherencia y racionalidad con la disciplina que requiere el método científico. El método a utilizar es la descripción que simultáneamente presenta concordancia con el diseño de investigación documental que explica Yuri y Urbano (2016) en los siguientes términos:

La investigación documental constituye una estrategia metodológica que emplea la investigación científica para realizar la revisión de los antecedentes de un objeto de estudio y para reconstruirlo conceptualmente.... permite contextualizar el fenómeno a estudiar, estableciendo relaciones diacrónicas y sincrónicas entre acontecimientos actuales y pasados (pág. 100)

De modo que, el diseño documental significa un tipo de estudio, que requiere descripción y comparación, se apoya principalmente en la búsqueda, recuperación, análisis e interpretación de fuente secundarios que provienen de fuentes documentales ya sean impresos, audiovisuales o electrónicos; mediante la recolección de información bibliográfica inherente al tema objeto de estudio, así como el registro organizado de la información para realizar el análisis, interpretación y utilidad práctica.

León Cedeño , P. U., \& Martillo Pazmiño , Ítalo O. (2020). Falsificación de marcas, competencia desleal y sanciones de la organización mundial del comercio. E-IDEA Journal of Business Sciences, 3(13), 12 -26. 
R E V I S T A

\section{RESULTADOS}

\section{Definición de Marca, Valor de la Marca y Funciones}

El comercio constituye uno de los factores primordiales en la dinámica de la actualidad internacional; de las operaciones de compra-venta dependen relevante cantidad de empleos, ingresos, logística e incentiva la producción de bienes y servicios; es frecuente que varias empresas concentren tiempo, esfuerzos y recursos en el diseño de la marca, de símbolos que contribuyen a identificar productos es la exhaustiva labor de marketing incluso los propios productos se convierten en emblemas de la marca por ejemplo: Mercedes Benz, Cristian Dior, Benetton, en el mercado existen una serie de símbolos, figuras, mensajes publicitarios que progresivamente contribuyen a identificar productos y crear las preferencias o el hábito de compra en los usuarios.

En este sentido, la premisa principal del artículo de investigación consiste en: ¿Cuáles son las consecuencias de la falsificación de marcas con la competencia desleal y las sanciones emanadas de la Organización Mundial del Comercio?

Una vez aclarado el método, se realiza la descripción de los principales conceptos inherentes al tema lo cual conduce al análisis de conceptos como marcas, valoración de la marca, competitividad, competencia desleal y sanciones; para efectos de mayor comprensión es pertinente detenerse en la definición de marca por Iglesias y Nicholas (2017) "la marca tiene que ver con los atributos tangibles e intangibles de un producto o servicio que ofrece una serie de significados para los consumidores".

Por consiguiente, la marca es la representación de bienes o servicios cuya características, calidad, precio, presentación y distribución satisfacen las expectativas de los compradores; cuando los usuarios encuentran el producto que corresponde a todos sus requerimientos las referencias son positivas, recomiendan el producto a otros potenciales clientes, se consolida el prestigio y confianza, así la marca logra el posicionamiento en el mercado; es el lugar que demuestra en el mercado el producto se evidencia en la percepción de los usuarios como el mejor producto de mayor calidad y óptima relación-precio valor, el diseño de la marca conduce a otra definición el valor de la marca por (Salinas, 2017):

Cuando hablamos del valor de marca nos referimos a valor económico financiero (monetario) y no a la apreciación subjetiva que un cliente o usuario puede realizar sobre una marca...las marcas crean valor impactando en las curvas de la oferta y la demanda. Por el lado de la demanda permite vender el producto a un precio superior dada la cantidad determinada de ventas (pág. 47) 


\section{E-IDEA}

R E V I S T A

J OURNAL OF BUSINESS SCIENCES

Para formular respuesta a la premisa inicial es pertinente la explicación de determinado conceptos tales como la competencia; es el dominio de capacidades, habilidades o destrezas para ejecutar determinadas actividades mientras que la competitividad se refiere la capacidad para la realización de actividades específicas los países son competentes cuando demuestran la capacidad para destacarse en las operaciones comerciales incluso cuando demuestran el nivel de especialización de alta calidad por ejemplo: Filipinas dispone de industria del calzado reconocida a nivel mundial, Francia destaca como el país que fabrica los cosméticos de más alta calidad e Italia se destaca por la fabricación de artículos de cuero, cabe considerar que Porter realiza una relevante innovación al explicar la competitividad desde otra visión en el sentido como él mismo lo afirma que la propiedad de los recursos y tecnología no siempre es una condición invariable para ser competitivo, ya que el hecho de utilizar y aplicar las estrategias de forma más práctica y eficiente contribuye a incrementar la producción e ingreso, por ejemplo: países como Singapur, Malasia, Filipinas tienen una óptima gestión del recurso humano aun cuando en todo los casos no e mano de obra calificada, pero si eficiente y productiva frente a Estados Unidos.

Por la propia naturaleza dinámica del comercio internacional las innovaciones tecnológicas son relevantes, contribuyen a la inclusión de cambios en los hábitos de compra de los clientes, en el cual influyen cultura y contexto en este sentido, la valoración de la marca es un exhaustivo trabajo de mercadeo en la cual destaca como estrategia de grandes empresas en la observación continua de las preferencias para comprender las causas que inciden en varios artículos tengan mayor demanda que otros como resultado del manejo de la publicidad.

En el contexto real, es muy difícil que en el mercado todos los factores se encuentren en igualdad de condiciones en relación a precio, presentación, distribución, calidad y marca en el estado de competencia perfecta ya que en ello influye desde la inflación hasta la accesibilidad al producto por la distribución, por ello lo más frecuente es la competencia imperfecta Berumen (2016) explica:

En un escenario de competencia imperfecta cada una de las empresas es consciente de que es capaz de afectar los precios, por lo cual la única manera de obtener mayores ventas será a través de la reducción de los precios de forma significativa, lo que conducirá a que cada una de las firmas se convierta en fijadora de precios.

En este orden de ideas, la diferencia se encuentra en la variedad de precios cuando las marcas de falsifican surgen una serie de aspectos como el nombre, presentación, empaque y logos son muy semejantes a la marca original, puede confundir a los clientes, por ello la

León Cedeño , P. U., \& Martillo Pazmiño , Ítalo O. (2020). Falsificación de marcas, competencia desleal y sanciones de la organización mundial del comercio. E-IDEA Journal of Business Sciences, 3(13), 12-26. 
estrategia consiste en la presentación del producto con leves cambios al original a un precio menor, por ello se define como competencia imperfecta o desleal.

La competencia imperfecta es una situación opuesta en la cual se destacan poca cantidad de oferentes, movilidad de mercancías, escasos factores productivos, libertades reducidas, no se conoce satisfactoriamente el mercado; sin embargo con la falsificación de las marcas, dicho enunciado no se cumple en su totalidad ya que surgen varios mecanismos de incumplimiento con los Tratados de Propiedad Intelectual e Industrial avalados por la Organización Mundial del Comercio; la falsificación es un delito que afecta la producción de las empresas con marca original.

Desde el año 2000, la falsificación de marcas se ha convertido en práctica frecuente, en la cual se aplican distintos métodos que están en contra de la esencia del Tratado de Propiedad Intelectual e involucra países como China, India, Singapur, Turquía y Emiratos Árabes Unidos como se explicara en la sección de resultados. La deslealtad se encuentra en el precio inferior en el mercado en relación a la marca original, aunque en principio se percibe como irrelevante, implica profundos cambios ¿Cuál es la reacción natural de los usuarios? Adquieren el producto que coincide con las expectativas de precio, calidad y presentación, paulatinamente desplaza la competencia del mercado, por ello es una práctica desleal.

Cuando se ofrecen productos a un precio inferior al de la marca original, la reacción natural de los usuarios es inclinarse a adquirir dicho producto, sin embargo, ello no garantiza el mismo nivel de calidad, certificación y permisos sanitarios, por lo cual implica un riesgo para el consumidor y disminución de rentabilidad para los productores.

\section{Falsificación de Marcas y Convenio de Propiedad Industrial e Intelectual}

La dinámica de la oferta y demanda, en el mercado implica la observación y análisis de varios factores, en los últimos 50 años muchas empresas han realizado una labor constante y continua, apoyándose en el marketing y publicidad para hacer de los productos y servicios símbolos e identificación de la marca misma. En dicha dinámica es frecuente que los usuarios asocien la calidad, presentación, valor del producto con la marca, así surgen las referencias y recomendaciones.

Cabe destacar que los productos son el resultado de la idea o creación intelectual de una persona, grupos de personas o de una empresa, dicha idea se perfecciona y amplia con el objetivo de satisfacer las expectativas de la población; la historia del marketing es abundante en muchos ejemplos: la creación de las muñecas Barbie fue una idea del matrimonio hebreonorteamericano Mattel en la búsqueda de un juguete agradable para su inquieta hija. 


\section{E-IDEA}

R E V I S T A

J OURNAL OF BUSINESS SCIENCES

Los creyones de cera, fue un experimento de Binney y Smith en 1903 con el propósito de desarrollar una nueva presentación de tiza de color no tóxica, posteriormente fue registrado con el nombre de "Crayola" el producto experimentó distintas innovaciones tanto en la presentación como en el uso de los materiales de 15 colores originales actualmente existe una variedad de 150 colores, también de ellos derivan los creyones en madera con cilindros de colores, aunque su elaboración es un secreto industrial también es un producto frecuentemente falsificado, especialmente en los países en vías de industrialización; pero ¿qué significa la falsificación de marcas? en una primera acepción por (Instituto Nacional de Propiedad Industrial (Inapi), 2018) opina que:

El término falsificación es de uso habitual en materia de Propiedad Industrial, para referirse especialmente al uso malicioso, con fines comerciales, de una marca igual o semejante a otra para los mismos productos, servicios o establecimientos. Entre los productos que pueden ser objeto de falsificación se cuentan: Ropa, Perfumes y cosméticos, Juguetes, Cigarrillos, Carteras y artículos de lujo Inapi, (2018)

Visto de esta forma, es pertinente profundizar en la interpretación del concepto la expresión 'uso malicioso, con fines comerciales' se infiere en el proceso de fabricación un factor que no está acorde con la normativa legal ya que por distintas razones se realizan leves modificaciones a logotipos, símbolos o el diseño mismo del producto, en ocasiones dichas diferencias son imperceptibles y desapercibidas por los usuarios, la distinción se encuentra en la calidad y el precio e incluso en los permisos sanitarios si son medicamentos o alimentos, en una segunda acepción la Agencia Tributaria citado por la Asociación General de Consumidores (2016) describe :

Se entiende por mercancía falsificada aquélla, incluido su acondicionamiento, en la que figure sin autorización una marca de fábrica o de comercio idéntica a la marca de fábrica o comercial registrada de forma válida para los mismos tipos de mercancías, o que no pueda distinguirse en sus aspectos esenciales de dicha marca de fábrica o comercial (pág. 1)

En este orden de ideas, la falsificación significa la fabricación con la inclusión de modificaciones de forma y presentación que alteran la marca original sin previa autorización, genera adversas consecuencias para la empresa propietaria de la marca ya que funciona como un engaño para los clientes que confunden el producto la apariencia, aspecto y presentación son muy semejantes, dichas diferencias casi imperceptibles tiene un efecto importante en el precio; el producto falsificado ofrece un producto semejante a menor precio, lo cual contribuye al declive de las marcas en el mercado y al desprestigio de la marca, además implican riesgos para la salud, propicia la explotación laboral, es un negocio ilícito que puede

León Cedeño , P. U., \& Martillo Pazmiño , Ítalo O. (2020). Falsificación de marcas, competencia desleal y sanciones de la organización mundial del comercio. E-IDEA Journal of Business Sciences, 3(13), 12 -26. 
asociarse con el crimen organizado. El uso de sustancias nocivas o tóxicas afectan a los trabajadores de estos recintos que generalmente no disponen de las normas mínimas de seguridad industrial.

Sin embargo a medida que el sector industrial, se extendió por todo el mundo también se crearon normas para garantizar el funcionamiento satisfactorio del mismo las cuales se expresan en el marco jurídico que incluye el Convenio de París para la Protección de la Propiedad Industrial (1883), el Convenio de Berna para la Protección de las Obras Literarias y Artísticas (1886) o Convenio de Berna, del cual emana posteriormente la Organización Mundial de la Propiedad Intelectual (OMPI) dicho marco jurídico fue concebido para proteger obras artísticas, literarias, científicas, fonogramas, emisiones de radiodifusión, invenciones provenientes de la actividad humana, descubrimientos científicos, diseños industriales, denominaciones comerciales, y marcas de fábrica de los plagios y falsificaciones; es inevitable preguntarse ¿Por qué si está disponible un amplio marco jurídico las falsificaciones son tan frecuentes?

Aún con un amplio marco jurídico, la ideas tienen una condición intangible no son palpables, son el núcleo de inicio para las creaciones artísticas y científicas a través de la mejora continua y el perfeccionamiento, en muchas ocasiones los propios creadores no tenían plena conciencia del significado del derecho de autor y la propiedad intelectual, por lo cual no se registraba la autoría de una obra debidamente, por esta razón es pertinente incluir el concepto de la (Organización Mundial de la Propiedad Intelectual (OMPI) , 2019)

El término que engloba las creaciones de la mente y la buena imagen comercial, aplicadas a los negocios, que pueden ser protegidas por la ley. La legislación de cada país difiere en cuanto a lo que la PI debe proteger exactamente, según el concepto que se tenga de los regímenes de PI. Ni el Convenio que establece la Organización Mundial de la Propiedad Intelectual (OMPI) ni el Acuerdo sobre los Aspectos de los Derechos de Propiedad Intelectual relacionados con el Comercio (Acuerdo sobre los ADPIC) definen directamente la propiedad intelectual (pág. 14)

En este orden de ideas, en el contexto del comercio internacional, los propios países debe propiciar las regulaciones acerca de la creación e invenciones para que las mismas no sean objeto de plagios, falsificaciones o versiones ilegales, o falsificación de marcas o productos está considerado un delito; por ello la Organización Mundial del Comercio apoyándose en la normativa previa ha creado convenios para sancionar y controlar dichas actividades, que han aumentado de manera preocupante en Asia, y son de importación frecuente en América las cuales se describirán en el siguiente apartado de Resultados. 


\section{E-IDEA}

R E V I S T A

J OURNAL OF BUSINESS SCIENCES

La Organización Mundial del Comercio (OMC) suscribe el Acuerdo sobre Aspectos de los Derechos de Propiedad Intelectual relacionados con el Comercio de carácter multilateral cuyo objetivo es la regulación en el comercio de conocimientos y contenidos creativos, vinculantes a la propiedad intelectual; los países miembros que se incorporen a dicho acuerdo asumen el compromiso de controlar las copias ilegales de artículos así como limitar el flujo de los mismos, China es el país en el cual se registran mayor cantidad de falsificaciones al respecto (Portafolio, 2019) aporta:

En términos relativos, lo que más se falsifica es calzado (el $22 \%$ ) del valor de lo aprehendido), ropa (16\%), productos de piel (13\%), material eléctrico (12\%), relojes (7 \%), equipamiento médico (5\%), juguetes (3\%), joyas (2\%) y fármacos (2\%) (Portafolio, 2019)

De acuerdo a la cita anterior, se interpreta que la industria china dispone de la tecnología y recursos para fabricar falsificaciones muy semejantes a los productos originales, por lo cual incrementa el precio y propicia mayor confianza y hábitos de consumo en los clientes; en pocas palabras es una 'imitación de calidad' a menor precio, lo cual motiva reacciones diferentes en los compradores.

Cabe destacar que de la propiedad intelectual; proviene la propiedad industrial que presenta dos clasificaciones: protección de signos distintivos de marcas de fábrica o comercio cuya función es la distinción de otras marcas y la indicación geográfica es la identificación del producto por su lugar de procedencia como un símbolo de reconocimiento. En el contexto de la Organización Mundial del Comercio, es un motivo de precaución constante la vinculación entre productos falsificados y crimen organizado, además que afecta las marcas comerciales y derechos de autor los grupos delincuenciales al falsificar productos evaden impuestos no generan empleos ni ingresos legales, por el contrario, propician la explotación laboral. Además, existen rubros de productos cuya calidad inferior puede ser perjudicial para los usuarios tales como medicamentos, productos farmacéuticos, eléctricos y cosméticos, al respecto la (Organización para la Cooperación y Desarrollo Económico (OCDE), 2019)

El informe de tendencias en el comercio de bienes falsificados y pirateados sitúa el valor de los productos falsos importados en todo el mundo, según los datos de incautaciones aduaneras de 2016, en 509.000 millones de dólares, frente a los 461.000 millones de dólares de 2013 (2,5\% del comercio mundial). En la Unión Europea, los productos falsificados representaban el 6,8\% de las importaciones de países no pertenecientes a la UE, frente al 5\% en 2013. Estas cifras no incluyen los productos falsificados producidos y consumidos a nivel nacional, ni los productos pirateados que se distribuyen a través de Internet OCDE (2019)

León Cedeño , P. U., \& Martillo Pazmiño , Ítalo O. (2020). Falsificación de marcas, competencia desleal y sanciones de la organización mundial del comercio. E-IDEA Journal of Business Sciences, 3(13), 12 -26. 
De lo anterior se destaca que, la constante copa de productos perjudica en gran medida las ganancias de las empresas que son dueñas de la marca original, estimula la competitividad desleal, contribuye a la desviación del comercio en términos formales y transparentes, desacredita la marca en el sentido de la calidad, resistencia y durabilidad. Además, se presenta el caso que se organizan rutas de transporte para los productos falsificados que involucran varios países que si bien no participan en la elaboración constituyen vía de tránsito para tal fin, lo cual también es parte del delito, continua ODCE (2019) en la explicación:

Los países más afectados por la falsificación en 2016 fueron los Estados Unidos, cuyas marcas o patentes se vieron afectadas por un $24 \%$ de productos falsificados incautados, seguidos de Francia, con un 17\%, Italia (15\%), Suiza (11\%) y Alemania (9\%). Cada vez son más las empresas de Singapur, Hong Kong y economías emergentes como Brasil que están entrando en el punto de mira. (Ob.Cit.)

Cabe destacar que el término Patente se refiere al registro formal en la autoridad nacional que señala como autor a la persona que ha realizado una invención creación u obra, en muchas ocasiones existen empresas que adquieren dicho documento para producir, perfeccionar y comercializar la creación; si el autor no registra el derecho como tal, las falsificaciones son muy difíciles de controlar; se requieren esfuerzos conjuntos entre varios países para tal fin, en ocasiones los controles en las aduanas son insuficientes, no corresponden a la secuencia de gestión y control satisfactoria, así como la capacitación del personal para detectar falsificaciones, progresivamente los productos se perfeccionan.

Aunque se estima que China es la economía que presenta mayor número de falsificaciones, se incorporan puntos de tránsito intermedio como Hong Kong, Singapur, Afganistán o Siria así lo acota Sandri (2016): “Las marcas más copiadas son Nike, Ray-Ban, Louis Vuitton y Rolex. Algunos consumidores pueden adquirir bienes falsos creyendo que son auténticos. La mercancía parece igual a la original (aunque no lo es) y el precio, muy similar".

Dentro de este marco falsificar consiste en introducir leves cambios en el diseño del producto por ejemplo: en el calzado se utiliza otros materiales en ocasiones de inferior calidad, se utiliza tonos de colores ligeramente más claros u oscuros, introducen pequeños cambios, muy leves que no sean obvios en el diseño, ya sean cambios en el nombre del producto, emblema, o logotipo que lo identifica, el precio no difiere significativamente, por lo cual los usuarios pueden creer que adquieren un producto original, la Oficina de la Propiedad Intelectual de la Unión Europea (EUIPO) (2021) aporta los siguientes datos: “2,5\% del comercio mundial en 2013 lo fue de productos falsificados y pirateados, lo que equivale a un 


\section{E-IDEA}

R E V I S T A

J OURNAL OF BUSINESS SCIENCES

máximo de 461 millones de dólares (EUR 338.000 millones de euros". De acuerdo a la cita anterior, en las dos últimas décadas se observa el creciente incremento de productos falsificados, cuya diferencias con el original son mínimas, como también se observa especialmente en las industrias alimenticias, farmacéuticas y de productos médicos, artículos que son 'versiones nacionales' de un producto original como lo es la marca de galletas Oreo, países como India, Brasil, Chile, Venezuela, Turquía entre otros fabrican el mismo concepto de galletas en ocasiones con nombres o presentación semejantes, de esta manera:

Los resultados muestran que, en 2016, las importaciones de productos falsificados y pirateados en la UE. Ascendieron a un total de 121000 millones de euros (134000 millones de dólares), lo que representa el 6,8\% de las importaciones de la UE, en comparación con el 5\% de importaciones de la UE de 2013 EUIPO (2021)

En este orden de ideas, en la organización de la Unión Europea se realizan importantes esfuerzos para evitar la distribución de productos falsificados en la región, sin embargo, considerando las cifras mencionadas los productos falsificados progresivamente han ingresado en el mercado europeo, desplazando en el mercado la producción regional y reduciendo las fuentes de empleo y posibilidades de inversión extranjera. Europa no está exenta del crimen organizado, que incursionan en la falsificación de marca, a lo cual se agrega la posición geográfica estratégica de Turquía; en medio de Europa y Asia, así lo explica (Caselles, 2017):

En Turquía se ha detectado el grupo criminal Bucak que controlaba en Kusadasi la venta de camisetas, camisas, pantalones, jerséis con marcas falsificadas mundialmente conocidas. El alcance del crimen organizado en las actividades de falsificación y piratería es amplio, cubriendo infinidad de sectores (pág. 64)

En poca palabras, la insuficiencia de control, escasa constancia y continuidad con el cumplimiento de la normativa acerca de Propiedad Intelectual, ha facilitado el desarrollo de flexibilidad que es sumamente riesgoso tanto para el equilibrio del comercio internacional, así como para la supervivencia de las empresas distribuidoras de marcas originales, aun cuando se establece la falsificación y piratería como delitos, la percepción que se tiene es que no se concientiza el carácter ilegal de dichas prácticas que incurren en hechos como el fraude es un mecanismo de engaño por que se utilizan símbolos y marcas para fabricar artículos sin autorización previa, ni patente de la empresa creadora.

En atención a lo planteado, por el hecho que la imitación de un producto es progresivamente perfeccionada hasta el nivel que no se diferencia significativamente del original, se evidencia un modelo de conducta modificado en los usuarios que se inclinan a

León Cedeño , P. U., \& Martillo Pazmiño , Ítalo O. (2020). Falsificación de marcas, competencia desleal y sanciones de la organización mundial del comercio. E-IDEA Journal of Business Sciences, 3(13), 12 -26. 
adquirir los productos falsificados, incluso a percibirlo como una actividad 'normal' desconociendo así todas las implicaciones y desventajas que genera a los creadores intelectuales, quienes son realmente los que pierden la regalía o utilidad que les corresponde; por último en la búsqueda de controlar dicha situación Estados Unidos ha creado el registro de los países en América Latina que fabrican mayores falsificaciones, tal como se presenta en la Tabla 1.

Tabla 1

Mercados de productos Falsificados:

\begin{tabular}{ll}
\hline País & Productos en Venta \\
\hline Argentina (Mercado la Salada) & $\begin{array}{l}\text { Falsificación de marcas en ropa y } \\
\text { calzado. }\end{array}$ \\
\hline Colombia (Mercado de San Andresito) & CD de música, películas y video juegos. \\
\hline Ecuador (Mercado Bahía de Guayaquil) & $\begin{array}{l}\text { DVD, CD, Programas de Computación, } \\
\text { ropa. }\end{array}$ \\
\hline México (Tepito y San Juan de Dios) & $\begin{array}{l}\text { CD,DVD, Video Juegos, equipos } \\
\text { electrónicos, ropa y calzado }\end{array}$ \\
\hline Paraguay ( Ciudad del Este ) & CD de música, películas y video juegos. \\
\hline
\end{tabular}

Fuente: (Sotomayor, 2021)

\section{CONCLUSIONES}

Una vez realizado la exhaustiva descripción del caso de falsificación de marcas se establecen las siguientes conclusiones: La Propiedad Intelectual e Industrial constituyen la protección hacia las ideas, como el primer concepto de la creación humana de la cual proviene las invenciones artísticas, científicas y literarias, una idea no es tangible, pero se expresa a través de la fabricación y diseño de objetos tangibles y comercializables dirigidos a satisfacer las expectativas de los usuarios.

Si bien los Convenios de Protección a la Propiedad Intelectual, no son de creación reciente, fueron aprobados a finales del siglo XVIII y significan la protección para los derechos de autoría es pertinente considerar que la propia innovación tecnológica se ha convertido en una importante herramienta para la falsificación de productos desde CD, equipos electrónicos, obras de arte, ropa y calzados; cuando se conocen y divulgan las técnicas para copiar e imitar con la inclusión de cambios imperceptibles para el usuario evidentes para el conocedor es una labor sumamente compleja controlar la falsificaciones.

No se evidencia en dicha actividad ventajas; por el contrario contribuye a la reducción de empleos, explotación laboral, propicia la propagación de delito organizado ya que existen 
grupos cuya ocupación consiste en fabricar falsificaciones generalmente para justificar la legitimación de capitales, pero el factor más importante el cual le atribuye la condición de delito es la falta de reconocimiento a la autoría de derechos así como concientizar que es una actividad ilegal; en América Latina se evidencia renuencia a aceptar dicha ilegalidad e incluso existen lugares comerciales que ofrecen estos productos en venta tal como se muestra en la Tabla 1.

La Organización Mundial del Comercio, a través de dos acuerdos, realiza la gestión de investigar, prevenir y controlar la difusión de artículos falsificados; sin embargo, intervienen otros factores como es la propia distensión de La Ley en los países fabricantes de falsificaciones en ello el mayor porcentaje se evidencia en China seguido de otros países como Singapur, Turquía, Emiratos Árabes u otros que constituye vías de tránsito como Afganistán o Egipto por lo cual la labor conjunta y el control constante es la estrategia más eficiente para prevenir las falsificaciones. 


\section{REFERENCIAS BIBLIOGRÁFICAS}

Asociación General de Consumidores. (2016). Asociación General de Consumidores, Estudios sobre el Consumo de Productos Falsificados. Publicación de la Cooperativa de Investigación Social y de Mercado. Versión Digital.

Berumen, S. (2016). Introducción a la Economía Internacional ESIC Editorial. Madrid, España : Edición Aumentada y Corregida.

Caselles, J. (2017). Derecho de Marcas y Proceso Penal. Madrid, España : Editorial Reus, Primera Edición.

Iglesias, O., \& Nicholas, I. (2017). Quiero esta marca!: Cómo construir marcas deseables. Barcelona, España : Servicios Editorial SCP.

Instituto Nacional de Propiedad Industrial (Inapi). (2018). Observancia, Piratería y Falsificación . Recuperado el 30 de Noviembre de 2021, de recuperado: https://www.inapi.cl.

Oficina de la Propiedad Intelectual de la Unión Europea (EUIPO). (2021). Tendencias en el mercado de Productos Falsificados y Pirateados. Recuperado el 28 de Noviembre de 2021, de https://euipo.europea.eu

Organización Mundial de la Propiedad Intelectual (OMPI) . (2019). Fundamentos de propiedad intelectual: Preguntas y respuestas para estudiantes. Recuperado el 06 de Diciembre de 2021, de https://www.wipo.int/

Organización para la Cooperación y Desarrollo Económico (OCDE). (2019). El comercio de productos falsificados representa ya un 3,3\% del comercio mundial, y va a seguir aumentando. Recuperado el 06 de Diciembre de 2021, de https://www.oecd.org

Portafolio. (2019). China sigue siendo el país en el que más se falsifican marcas . Recuperado el 06 de Diciembre de 2021, de https://portafolio.co

Salinas, G. (2017). Valoración de marcas: revisión de enfoques, metodologías y proveedores. Barcelona, España. : Ediciones Deust.

Sampieri, R., Fernández, C., \& Baptista, M. (2019). Metodología de la Investigación. México D.F.: Mac Graw Hill Interamericana Editores. Sexta Edición.

Sandri, P. (2016). Las copias falsas ya representan el 2,5\% del comercio mundial. Recuperado el 28 de Noviembre de 2021, de https//www.lavanguardia.com 
Sotomayor, A. (2021). EEUU publicó su lista negra de mercados piratas en el mundo. Recuperado el 03 de Diciembre de 2021, de Https://www.legiscomex.mx

Yuri, J., \& Urbano, C. (2016). Técnicas para. Recursos Metodológicos para la Preparación de proyectos de Investigación. Córdoba, Argentina: Editorial Brujas, Volumen 2, Segunda Edición. 